\title{
Bladder leiomyoma: Lower urinary tract symptoms with an unusual finding
}

\author{
Joseph Panza $^{1 *}$, Tiffanie Tam ${ }^{2}$ Jeffrey Segal $^{3}$ and Stuart Shoengold ${ }^{3}$ \\ ${ }^{1}$ Department of Obstetrics and Gynecology, Vanderbilt University Medical Center, Nashville, TN, USA \\ ${ }^{2}$ Department of Obstetrics and Gynecology, Trihealth, Cinncinati, OH, USA \\ ${ }^{3}$ Department of Obstetrics and Gynecology/ Department of Urology, Saint Barnabas Medical Center, Livingston, NJ, USA
}

\begin{abstract}
Background: Mesenchymal tumors of the bladder encompass 1-5\% of all bladder tumors. Bladder leiomyomas encompass this subset and are generally uncommon. These benign tumors are frequently diagnosed via routine ultrasound and biopsy should be performed in order to rule out malignancy.

Case: We present a case of a 56-year-old woman with various lower urinary tract complaints for 6 months duration. After imaging, she was found to have a bladder mass which was resected and found to be a leiomyoma on final pathology.

Conclusion: Bladder leiomyoma is a rare finding and commonly presents with symptoms of urinary obstruction or pain. Most can be treated with resection and overall prognosis is good with an estimated $18 \%$ recurrence rate.
\end{abstract}

\section{Introduction}

Leiomyoma of the bladder is a rare mesenchymal tumor with about 250 cases reported [1]. Goluboff et al. reported that leiomyoma of the bladder occurs most frequently in the 3 rd through $6^{\text {th }}$ decade of life, with an average age of 44 years old [2]. It occurs predominantly in females $(76 \%)$, however, there are cases that have documented males and children. Patients present primarily with symptoms of obstruction, but can also have associated dysuria, hematuria, and pelvic pain. The location of the tumors have been reported intra-vesical (63\%), extra-vesical (30\%) or intramural (7\%) [3]. Most common imaging modalities include US, but CT and MRI can also be used to help delineate the location of the tumor, as well as characteristics to rule out leiomyosarcoma and additional bladder malignancies.

Overall prognosis is good after surgical resection, which can be performed by a trans-vaginal approach or transurethral resection for smaller endo-vesical tumors.

\section{Case Report}

The patient is a 56-year-old post-menopausal female with past medical history of arthritis, GERD, obesity, and peripheral vascular disease presenting with a history of urinary urgency, frequency and nocturia for a six-month period. She was initially evaluated by her primary OBGYN, at which time a transvaginal ultrasound revealed a normal appearing post-menopausal anteverted uterus, with a 1.3 $\mathrm{cm}$ anterior wall intramural fibroid, $4 \mathrm{~mm}$ endometrial lining and a $4.2 \times 3.9 \times 2.5 \mathrm{~cm}$ solid bladder mass originating from the left wall (Figure 1).

Recommendations for cystoscopy and MRI were given by the radiologist. Patient was then referred to urology for follow up.

Initial urologic work up including complete blood count, liver and kidney function tests, urinalysis, urine cytology, and urine FISH showed no abnormalities. The patient underwent renal and bladder US, which showed bilateral ureteral efflux and a $4.57 \mathrm{~cm}$ tumor on the left bladder wall without other abnormalities (Figure 2). CT scan of the abdomen revealed no hydronephrosis, hydroureter, or enlarged lymph nodes. The decision was made to take the patient for cystoscopy and biopsy with possible transurethral removal of the mass. Cystoscopic findings revealed a golf ball-sized solid tumor that appeared prostate-like. The mass extended from the floor of the bladder covering the left orifice.

The etiology of the mass was unclear as was whether or not it was invasive in nature. The right orifice appeared normal. A resectoscope was used to remove the mass without ureteral stent placement.

Pathology of the mass describes benign urothelium with underlying large fragments of smooth muscle consistent with leiomyoma. Staining for Ki-67 proliferation marker was negative and smooth muscle antigen was strongly position confirming a benign smooth muscle mass.

The patient was placed on Vesicare for urinary frequency and antibiotics for post-operative UTI prophylaxis. She returned to the office within one week of the procedure with continued complaints of urinary frequency and urgency and was maintained on the Vesicare. One month later, the patient underwent repeat renal and bladder US, which was without evidence of residual bladder mass. The patient's symptoms of urinary frequency had improved. Repeat cystoscopy three months after the primary procedure revealed bladder scarring along

${ }^{*}$ Correspondence to: Joseph Panza, Department of Obstetrics and Gynecology, Vanderbilt University Medical Center, Nashville, TN, USA, Tel: 203-768-1796; E-mail: joseph.r.panza@vumc.org

Key words: bladder leiomyoma, mesenchymal tumors, bladder tumors, urinary obstruction

Received: January 10, 2019; Accepted: January 25, 2019; Published: January 28, 2019 


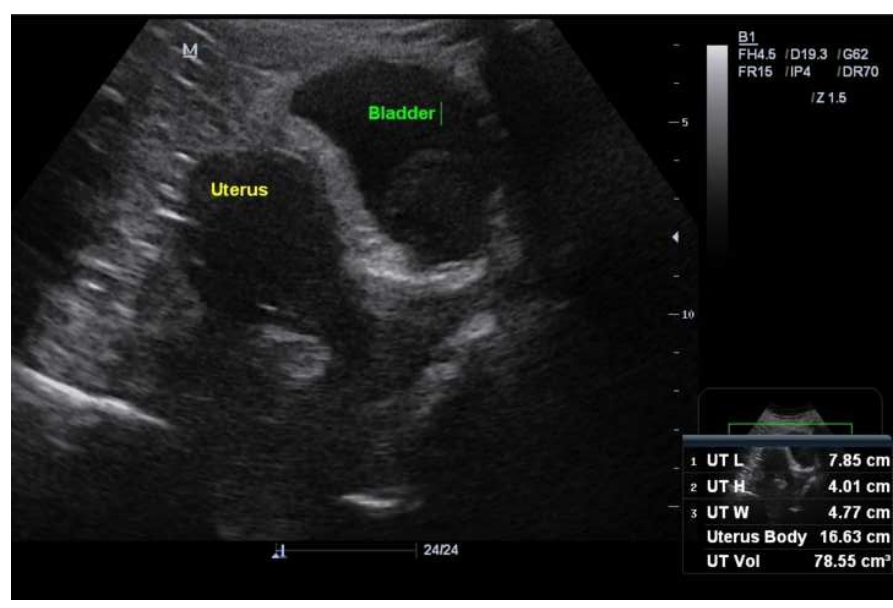

Figure 1. Ultrasound revealing mass within bladder and adjacent uterus

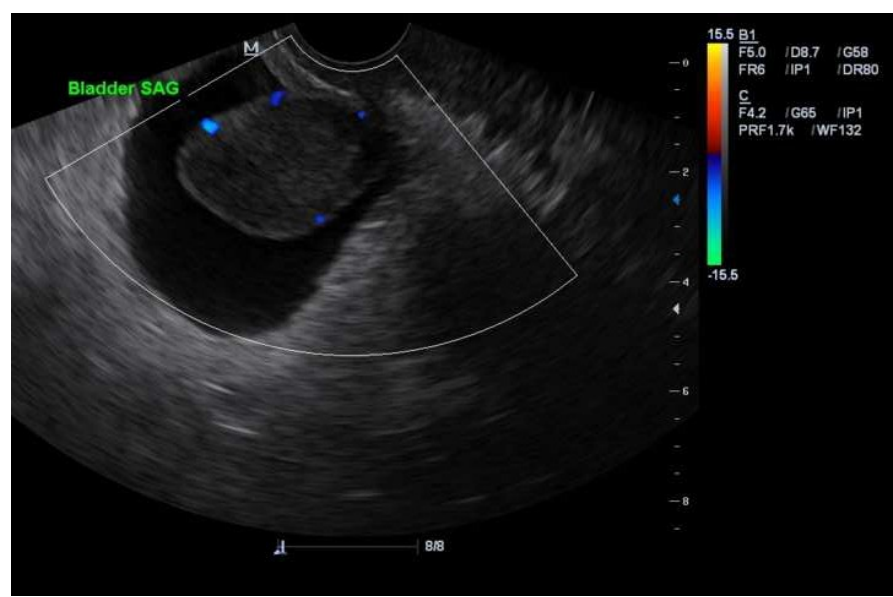

Figure 2. Ultrasound with sagittal view of bladder mass

the prior resection site, but no residual mass. Biopsies of the prior area of resection were taken and pathology revealed fragments of smooth muscle bundles showing areas of active acute and chronic inflammation with degenerative changes. The patient had improved lower urinary tract symptoms upon removal of the mass and follow up cytoscopy was scheduled for six months.

\section{Discussion}

Mesenchymal tumors represent 1-5\% of all urinary bladder tumors. Leiomyomas encompass $35 \%$ of this subset and $0.43 \%$ of all bladder tumors [1]. It is a rare differential diagnosis, however, and should be confirmed by biopsy and histological evaluation prior to surgical management. It is important to rule out bladder pathology such as leiomyosarcoma or inflammatory myofibroblastic tumor. Most patients will present with obstructive symptoms, dysuria, hematuria or it may be found as an incidental finding on imaging. That being said, other presenting symptoms such as urinary urgency and frequency, as with our patient, may also be present. Commonly, US is used as the primary imaging modality, however, an MRI may be useful in determining location or the tumor, depth of invasion, size as well as assessing areas suspicious for malignancy.

Etiologies of bladder leiomyomas are unclear. It is hypothesized that it may be driven by estrogen due to case reports of estrogen receptors found on pathology [2]. There has also been an association of pulmonary lymphangioleiomyomatosis in patients who later present with bladder leiomyoma [4]. Treatment of bladder leiomyoma depends on the location, size and symptoms of the patient. If the patient is asymptomatic, and biopsy has confirmed leiomyoma, it is plausible to wait and repeat imaging in a later date. For patients who are symptomatic with small bladder leiomyomas, transurethral resection can be performed safely, however it has been documented that $18 \%$ of cases may need re-operation [1]. These were removed by TUR and likely due to incomplete resection. Larger leiomyomas may require an open procedure with partial cystectomy. Overall prognosis for bladder leiomyomata is good after surgical resection and recurrence has been documented in only few cases.

\section{References}

1. Hater N, Sakr G (2013) Bladder Leiomyoma: Presentation, evaluation, and treatment Arab J Urol 11: 54-61. [Crossref]

2. Goluboff ET, O'Toole K, Sawczuk IS (1994) Leiomyoma of bladder: report of case and review of iterature. Urology 43: 238. [Crossref]

3. Jain S, Tanwar R, Mitra A (2014) Bladder leiomyoma presenting with LUTS and coexisting bladder and uterine leiomyomata: A review of two cases. Rev Urol 16: 5054. [Crossref]

4. Yin F, Wang N, Wang Y, Bi X, Xu X, et al. (2015) Transvaginal Resection of a Bladder Leiomyoma Misdiagnosed with a Vaginal Mass: A Case Report and Literature Review. Case Rep Obstet Gynecol 2015: 981843. [Crossref]

Copyright: (C2019 Panza J. This is an open-access article distributed under the terms of the Creative Commons Attribution License, which permits unrestricted use, distribution, and reproduction in any medium, provided the original author and source are credited. 